\title{
Estrategias dirigidas a las atenciones de salud domiciliaria
}

\author{
Dayvis Tomas Barreto Minaya \\ 1807barreto@gmail.com \\ Fernando Emilio Escudero Vílchez \\ fescudero@silfersystem.com \\ Silvia Liliana Salazar Llerena \\ Ssalazar@silfersystem.com \\ Universidad César Vallejo \\ Lima - Perú
}

\section{RESUMEN}

OBJETIVO: Como fin tuvo presentar las diferentes estrategias que están dirigidas a la salud pública a domicilio e identificar las perspectivas de otros autores acerca de ello. METODOLOGÍA: enfoque cuantitativo, diseño descriptivo y una revisión sistemática, para el cual se revisará la normativa vigente y se analizarán los pros y principales problemas en el sector salud, para establecer soluciones significativas que ayuden a la mejora del ámbito salud en la región Ancash, de la mano con la satisfacción del usuario. RESULTADOS: Al ser una investigación sistemática está basada en revisión bibliográfica y análisis empírico sobre las principales problemáticas del sector salud. CONCLUSIÓN: Además, brinda una alternativa de solución a través de las visitas domiciliarias, componente importante en la promoción de la salud, la cual buscan justamente conocer los posibles males de la población en sus etapas iniciales y establecer políticas para que estos sean atendidos y generar una solución eficaz en el bienestar de los pacientes y población vulnerable.

Palabras claves: estrategia; atenciones de salud; sistema de salud 


\title{
Strategies aimed at home health care
}

\begin{abstract}
OBJECTIVE: The aim was to present the different strategies that are aimed at public health at home and to identify the perspectives of other authors about it. METHODOLOGY: quantitative approach, descriptive design and a systematic review, for which the current regulations will be reviewed and the pros and main problems in the health sector will be analyzed, to establish significant solutions to help improve the health sector in the Ancash region, hand in hand with user satisfaction. RESULTS: Being a systematic research, it is based on a literature review and empirical analysis of the main problems in the health sector. CONCLUSION: In addition, it provides an alternative solution through home visits, an important component in health promotion, which precisely seeks to know the possible ailments of the population in their initial stages and establish policies so that these are attended and generate an effective solution in the welfare of patients and vulnerable population.
\end{abstract}

Key words: strategy; health care; health system.

Artículo recibido: 15 noviembre. 2021 Aceptado para publicación: 10 diciembre 2021

Correspondencia: 1807barreto@gmail.com Conflictos de Interés: Ninguna que declarar 


\section{INTRODUCCIÓN}

Actualmente, Such et al. (2020) consideran que el sistema de salud público no ofrece un servicio de calidad ni estrategias gubernamentales que faciliten la atención a los pacientes, lo cual ocasiona que estos no puedan acceder de manera óptima a los diferentes servicios. El sistema de salud, como muchos otros, ha pasado por una serie de "mejoras", las cuales muchas veces fueron dedicadas a un sector en específico, siendo perjudicial para el desarrollo común de la sociedad (Smith et al., 2020).

Para el autor Staniforth \& Such (2019), las entidades pertinentes han enfrentado problemas y aplicado estrategias de la solución a los problemas, con el fin de buscar la satisfacción de los pacientes, sin embargo, aún existen insuficiencias; es así que, las diferentes direcciones de salud han manejado este tema de formas distintas, ocasionando que los resultados no sean los mejores y acrecentando los problemas ya existentes como las demoras en los trámites administrativos, la mala atención, la escasez de medicamentos, la sobredemanda y otras dificultades, generando insatisfacción en los pacientes o usuarios finales, quienes son los más afectados.

Currie et al. (2020) refiere que la salud pública se encuentra en condiciones desalentadoras debido a la falta de una gestión pública responsable orientada al cambio. El ámbito de la salud debe estar regido por lineamientos que permita su desarrollo y el de la sociedad, de manera sostenible y equitativa. Por tal motivo, el autor Zhang \& Nie (2021) demuestran que la salud debe caracterizarse por ofrecer servicios de calidad, crear estrategias que priorizan al sector y organice los procesos que se necesitan realizar, debido a que muchas veces estos no son aplicados de manera correcta, siendo ineficaces a la hora de lograr las metas planteadas.

Da Silva et al. (2018) precisa que un sistema de salud óptimo es parte fundamental de la gestión pública realizada por los representantes de la comunidad, siendo estos los que toman decisiones para mantener las necesidades de la sociedad cubiertas. El personal de salud, los implementos de seguridad, los medicamentos y todas aquellas herramientas fundamentales en los centros de salud deben cumplir con la calidad y ser supervisados dentro del marco legal. Sin embargo, en América Latina los sistemas de salud no se encuentran adecuadamente desarrollados por la existencia de dos frentes, el sector público, representado por el Estado y el sector privado, que lo conforman entidades independientes. Esta división a lo largo de los años ha generado que exista una 
desigualdad para el acceso a la salud, ya que en el sector privado se observa que los servicios no son los adecuados, siendo este el importante en una sociedad.

Así mismo, Rossetto et al. (2020) relaciona los problemas en el sector salud actual con la corrupción, debido a que los recursos públicos no se emplean de la manera adecuada, destinando el dinero para satisfacer las necesidades privadas y no colectivas. No se trata de falta de recursos, sino de un mal manejo por la desviación de los mismos. Por otro lado, también precisa que el incumplimiento es un factor importante, porque las normas que regulan al sector no son supervisadas. Cabe señalar que los conflictos del Estado y el ámbito privado también influyen de manera negativa, debido a que se priman los intereses de un sector en particular.

En cuanto al Perú, se ha implementado políticas en el área de salud junto a alianzas que permiten el desarrollo de la sociedad, como pensión 65, Cuna más y Qali Warma, los cuales ayudan a las familias en zonas rurales que se encuentran en situación vulnerable, permitiendo descentralizar la atención y protección nacional. Es así que nacen estrategias y metas multisectoriales que abarcan varios sectores del Estado, luchar y minimizar los problemas existentes en el país, como la anemia, las brechas en la salud, el déficit en educación, etc. Todas estas estrategias tienen las características de ser medibles en el tiempo, visibilizar su efectividad y posibles mejoras, de acuerdo a las entidades involucradas como lo son el Ministerio de la Mujer y poblaciones vulnerables, Ministerio de desarrollo e inclusión social, etc.

Es así que se considera importante que exista un compromiso por parte de las autoridades en conocer verdaderamente el estado en el que se encuentran los servicios públicos para plantear mejoras y estrategias que aligeren la sobredemanda, teniendo en cuenta a todos los grupos sociales que necesitan este servicio básico (Fan et al., 2021).

\section{ESTRATEGIAS METODOLÓGICAS O MATERIAL Y MÉTODOS}

El presente trabajo presenta un enfoque metodológico donde se adjunta y contrasta la información para verificar que sea la adecuada según los buscadores seleccionados. Por ello, se expondrán dos esquemas, el esquema de revisión y el de PRISMA, para dar una mejor organización en relación a las referencias de las fuentes escogidas.

\section{Tipo y diseño de investigación}

En la investigación se utilizó la variable "Estrategias de las atenciones de salud” y puntualizó como objetivo general presentar las diferentes estrategias que están dirigidas 
a la salud pública a domicilio, con el fin de dar conocimiento sobre el tema y que tan influyente es en las personas. Es decir, la variable fue escogida para determinar el efecto que efectúa en otras, con el objetivo de identificar la relevancia en la realidad del trabajo (Batt \& Kahn, 2021).

De la misma forma, la revisión sistemática recopila, indica y estima de manera crítica para que después se realice un resumen sobre el tema determinado (Moreno et al., 2018).

\section{El esquema de la revisión de artículo y su desarrollo se presentarán a continuación:}

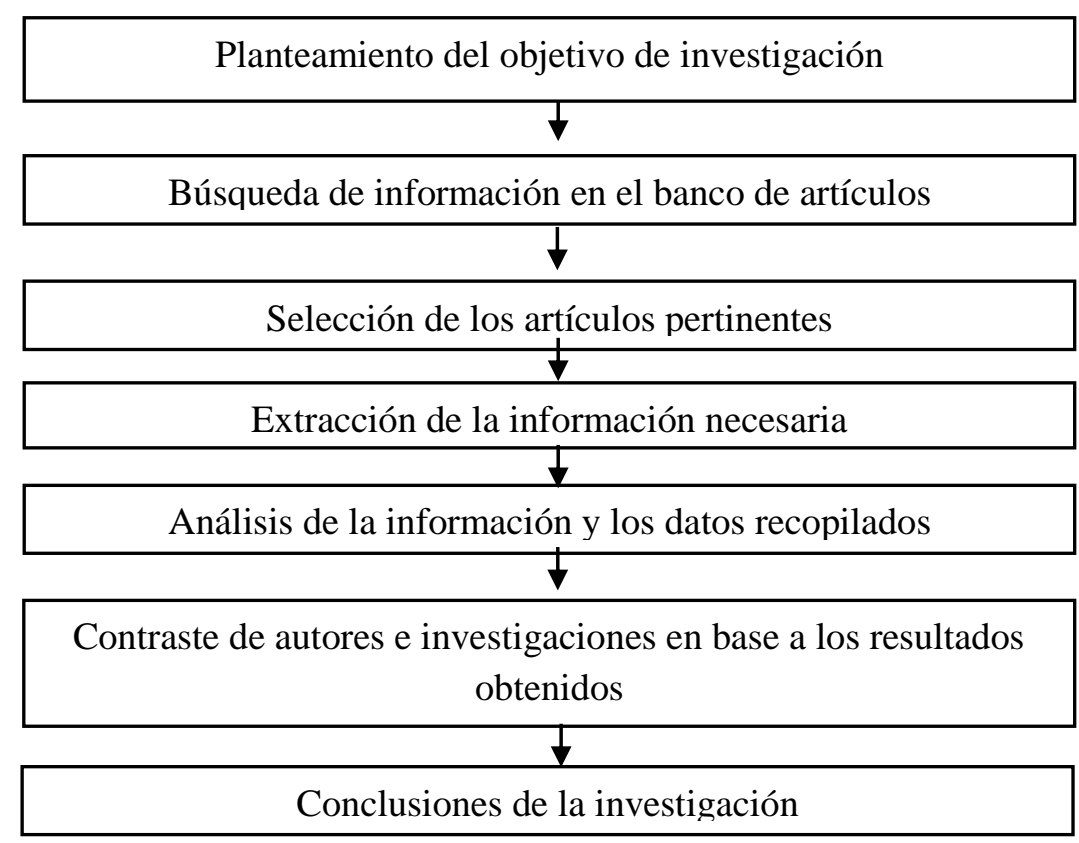

Figura: Esquema de la revisión del artículo

\section{Técnicas e instrumentos de recolección de datos}

En el presente trabajo se realizó de forma efectiva ya que se recopiló varios artículos acerca de las diferentes estrategias en la salud pública; es así que, se tuvo como fuentes confiables a los artículos de revistas indexadas, con el fin de que los datos encontrados sean de diferentes perspectivas.

Se presentaron los siguientes filtros para poder escoger los artículos que tengan características similares:

- Dentro del periodo 2017-2021.

- En el idioma inglés (98\%) y español (2\%)

- En relación a la variable o el título.

- Los resultados y conclusiones se contrastan al trabajo.

- Perspectivas diferentes por parte de los autores y países. 
- Revistas indexadas fiables.

La metodología del estudio se inclina por el paradigma cualitativo, el cual describe una realidad social, descriptiva, según señalan que la investigación cualitativa se centra en comprender y profundizar los fenómenos, explorándolos desde la perspectiva de los participantes en un ambiente natural y en relación con el contexto de investigación para que sea de utilidad en futuras disposiciones (Maeda et al., 2020).

\section{Procedimiento}

Los datos mostrados son por medio de la revisión sistemática y bibliografía; además, el criterio de inclusión fueron los años de publicación, el contenido, la realidad y los resultados de los autores.

Además, se hizo uso de buscadores de internet como Scielo, Scopus y Google Académico, en ellos se pusieron las palabras clave que fueron "salud pública y "estrategias de atención a la salud".

Las investigaciones seleccionadas se caracterizaron por ser en su mayoría de lengua inglés y español, aparte, aquellos que mantenían una relación con la variable de estudio o que de alguna concordancia con los resultados que se querían obtener. En el buscador Scielo se encontró artículo que tenía todos los requisitos requeridos, siendo 5 en total, también para Scopus fueron 20 artículos.

Los artículos para ser incluidos tuvieron que mantener un perfil estricto; por ejemplo, que sean de revistas indexadas conocidas y fiables, con objetivos similares a la investigación, resultados contribuyentes e importantes. Asimismo, los que presenten variedad de opinión por los diferentes autores para tener una visión más amplia y clara.

Dicho esto, los descartados para usarlos en la investigación fueron aquellos que no proporcionaban con la palabra clave, artículos incompletos porque no tenían resultados o conclusiones, tampoco los que no mostraban una bibliografía eficiente, estudios que no mantenían relación con el trabajo y que no estén en el tiempo o con el idioma requerido. Por consiguiente, se efectuó en tres filtros: el primero están los artículos que son parte de los buscadores presentados, el segundo por el título y el tercero fue la información obtenida de su metodología, resultados y conclusiones.

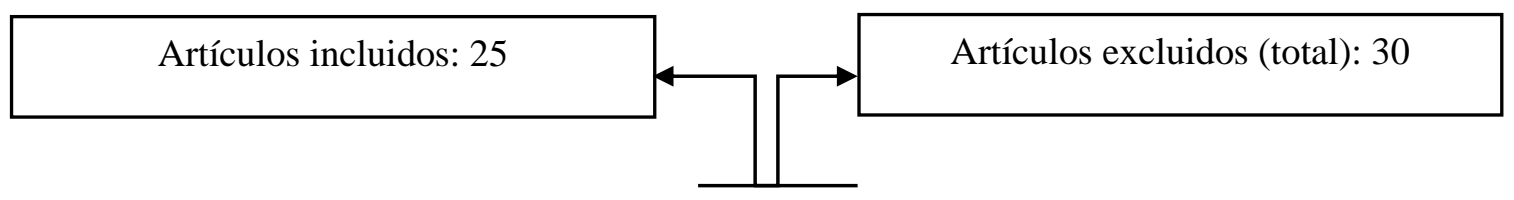




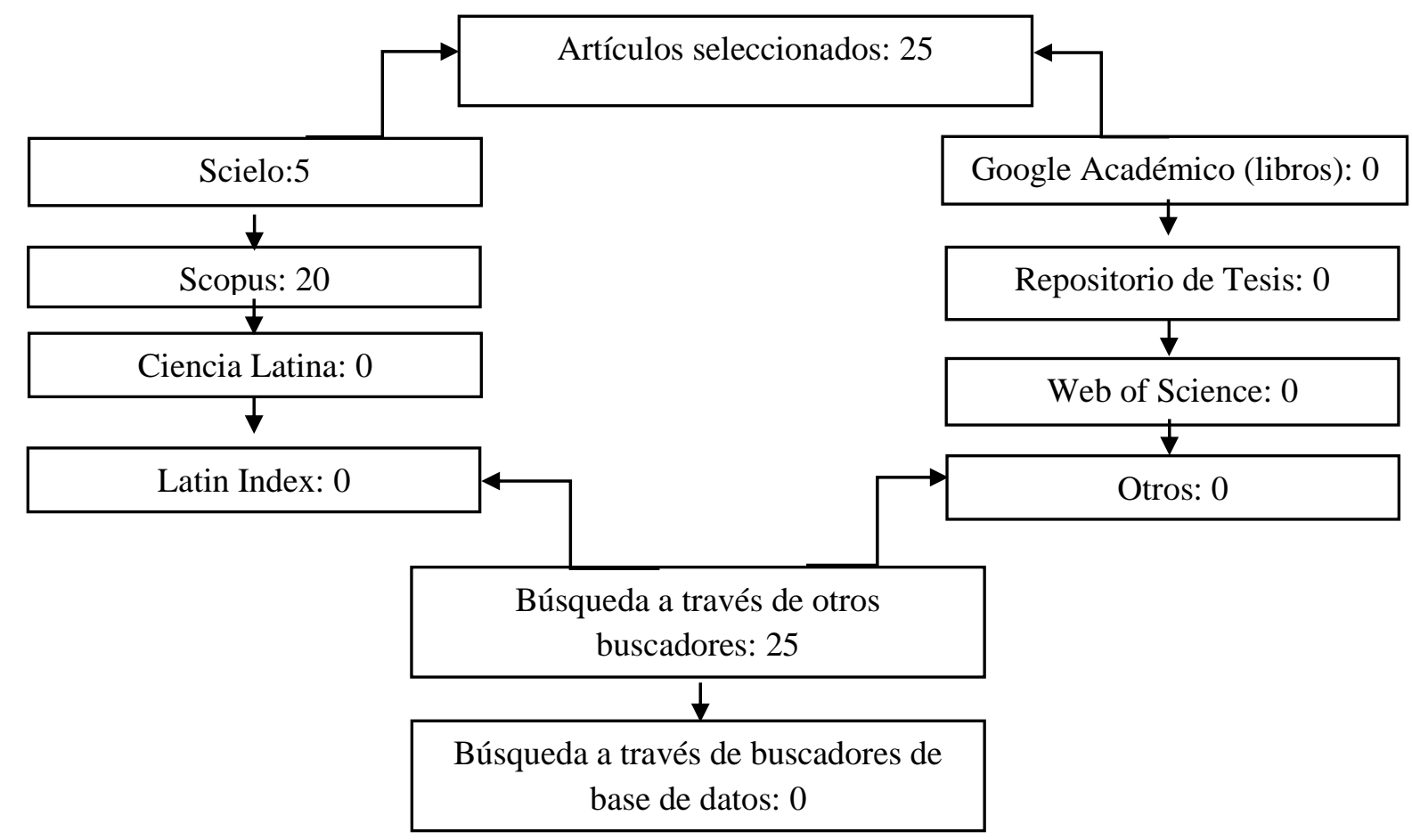

Figura: Esquema PRISMA

\section{RESULTADOS Y DISCUSIÓN}

Los autores Ritchie et al. (2021) resaltaron que la atención domiciliaria parte como una manera de atender las demandas de una población minoritaria, facilitando las acciones del paciente. La mayor parte se encuentra compuesta por adultos de la tercera edad, pacientes crónicos o una población con limitaciones físicas. Igualmente, Wendt et al. (2021) identifica a este tipo de servicio, más que por un tema de comodidad, en muchos casos se da por seguridad, porque es necesario brindarles un apoyo instrumental extra y una atención más personalizada, de sobre manera en los casos de cuidado como pacientes ancianos dependientes. Los autores revisados en este trabajo, siendo uno de ellos Becker (2017), afirmaron que las visitas domiciliarias permiten brindar orientación y atención personalizada a los pacientes, lo cual es sumamente importante para aliviarlos y cuidar de ellos, ya que son la población de riesgo y no pueden hacerlo por sí mismos.

\section{Discusión}


Para el autor Lim et al. (2021) manifestó que la visita domiciliaria no es aplicable en todos los casos, solo en aquellos que se tenga a personas mayores de 65 años porque en ellos el acudir presencialmente a centros médicos podría atentar contra su fragilidad, ya que su sistema inmunológico no es tan fuerte al de una persona más joven.

La falta de movilidad de estos ciudadanos hace presente la necesidad de un medio personalizado para su tratamiento, de la misma manera se encuentra que, como medio de prevención, realizar visitas que llevan el control del estado de salud del paciente puede ser una alternativa bastante eficiente (Wanaratwichit et al., 2019).

Según Ngan et al. (2021) la atención domiciliaria ha sido una herramienta importante tanto en el control y prevención durante la pandemia por el virus Sars-CoV-2, lo cual permitió evitar las aglomeraciones en los centros de salud y apoyar de manera personal a la atención de pacientes con tratamientos especiales, los cuales no podían acudir a una atención medica presencial por cuestiones de seguridad. En la misma línea, Kiernan \& Kilbride (2021) consideran que en este caso las visitas permitieron que estos ciudadanos puedan seguir teniendo intervenciones y controles, y de la misma manera se atendieron emergencias leves, evitando contagios, debido que los profesionales de la salud realizaban las visitas correctamente protegidas, siguiendo las medidas de seguridad obligatorias.

Por su parte, Munhak (2020) indica que las visitas domiciliarias brindan comodidad y beneficio para los ciudadanos mayores, siendo estos los que se sienten más seguros al evitar acercarse a los establecimientos de salud por la presencia de enfermedades que podrían contraer. Si bien por una parte es un beneficio notorio para cierto grupo específico, algunas veces esta herramienta de ayuda no puede ser realizada de manera adecuada debido a la falta de personal para cubrir la demanda de los pacientes que sí pueden acudir de manera presencial. Se estima que alrededor del $30 \%$ del presupuesto designado al ámbito salud se ve afectado en mantener este sistema, retrasando acciones de crecimiento y desarrollo institucional (Powers et al., 2021).

Los autores Si-qian et al. (2021) precisaron que los ciudadanos que llevan una enfermedad mental en proceso de tratamiento tienen cambios positivos al desarrollar su atención médica en casa, puesto que un ambiente familiar cómodo permite reducir las consecuencias negativas. En el caso de los pacientes atendidos, de la población total (638), los pacientes con gran número de atención fueron aquellos con demencia, siendo 
el 30,7\%. En ellos se pudo notar diferencias positivas en cuanto a su estado de salud cuando seguían un tratamiento presencial, debido a que se presentaba una mayor cuantía del síndrome de inmovilidad (70,3\% contra el $24 \%)$.

Yehenew \& Weihua (2018) estima que, a raíz de los problemas en el sistema de salud, las visitas domiciliarias se convierten en una opción importante en el componente preventivo promocional, además permite una aproximación de las familias y el sistema de salud, pues pueden acceder a los servicios básicos que por ley les corresponde. En otros países es un instrumento que se usa desde hace muchos años y ha traído resultados positivos, porque permite que el personal pueda llegar a zonas alejadas y a personas que no pueden acudir por sí mismos a los centros de salud.

Por otro lado, Vandan et al. (2020) reporta que las visitas domiciliarias son establecidas en pacientes que presentan mayor número de patologías, pertenecientes a la zona rural y zona urbana. Los profesionales de enfermería acuden con una frecuencia limitada a supervisar a los pacientes, a quienes se les ofrece un trabajo personalizado y de mayor cuidado, ya que es dirigido a personas mayores de 65 años con enfermedades que podrían ser mortales si no se lleva un control responsable; aparte, estas visitas permiten atender las demandas de personas que no podrían acceder a este beneficio por encontrarse en situaciones precarias, por tal motivo se busca satisfacer sus necesidades, sin incurrir en el incremento de costes de la atención (Videon et al, 2021).

De la misma manera, Pailliard et al. (2019) indicaron que la mejora del servicio de atención es mucho más notoria al ser personalizado y de manera domiciliaria, ya que 70 pacientes se beneficiaron con esta alternativa debido a que llevaban un tratamiento paliativo. Si bien los resultados son desalentadores al conocer el deceso de los pacientes, resulta importante señalar que el cuidado fue lo primordial para los ciudadanos. Alrededor del 71,4\% falleció en el hogar, de los cuales un 92\% pudo elegir conscientemente que este hecho sucediera en casa. Estos datos permiten evidenciar la importancia de que pacientes con tratamientos oncológicos, como es el caso del 88,6\% de personas en este estudio, puedan acceder a un cuidado total y responsable. La falta de la capacidad de manera autónomo es un hecho frustrante para estos individuos, por lo que la presencia de un soporte externo puede ser clave para una estadía próspera.

\section{CONCLUSIONES O CONSIDERACIONES FINALES}


Se pudo concluir que no existe en el sector salud un sistema estatal infalible para poder minimizar un ataque epidemiológico de esta magnitud como lo es la SARS-COV-2, ya que la pandemia ha hecho que todos los gobiernos replanteen su gestión pública e implementen estrategias adecuadas para combatir la pandemia. El sector salud es sumamente importante en una sociedad, por tal motivo debe tener las facilidades para actuar en caso la situación lo amerite, así como acceder a información pertinente que los pueda orientar y brindar soporte.

En el Perú, en primer lugar, el desconocer cómo operan las distintas entidades gubernamentales no ha ayudado a crear nuevas políticas públicas para mitigar el daño causado por SARS-COV-2, además de la escasez de recursos de previsión. El país ha sido afectado por esta pandemia en todos los ámbitos, agudizando los problemas existentes en la administración pública nacional, sumado a esto el desorden, el miedo a la enfermedad y la inconformidad a los planes de acción, así como la crisis política que se presenció. El país se encuentra en un desorden interno de gran magnitud, ha podido resolver algunos problemas, pero ha dejado a otros de lado, ocasionando que la sociedad actual se vea afectada.

Las personas en una sociedad democrática eligen a sus representantes para que estos puedan abogar por ellos y administrar de manera óptima los recursos nacionales, sin embargo, el dinero destinado al bienestar social no es correctamente empleado. Los ciudadanos deben exigir a sus autoridades que se aumente el nivel de calidad de los recursos y que se implementen estrategias de auxilio, ya que la pandemia ha demostrado de manera mundial que las personas no estaban listas para enfrentar un virus mortal. Los números de víctimas a raíz de la SARS-COV-2 fueron terribles, y si el Estado no administra los recursos y toma acción, en caso suceda otro acontecimiento de tal calibre, los números se tripliquen.

Finalmente, las visitas domiciliarias son una estrategia que ayuda de manera significativa en identificar de manera temprana las características en salude las familiar y además dar un mejor acercamiento a las posibles causas de los males con los que padecen sus integrantes, información de relevancia para las estrategias preventivo promocionales en salud, pero de todas maneras no se debería perder el enfoque en el sistema de salud, ya que es el servicio más importante en la actualidad 
y descuidarlo por empezar a realizar una apuesta total en el establecimiento de la atención domiciliaria sería un retroceso en las mejoras desarrolladas.

\section{LISTA DE REFERENCIAS}

Batt, R., \& Kahn, L. (2021). Data Transparency and Methods in Quantitative and Qualitative Research. The Journal of Work and Policy, 74(5), 1079-1082. https://search.ebscohost.com/login.aspx?direct=true \&db=edshol\&AN=edsho 1.hein.journals.ialrr74.65\&lang=es\&site=eds-live

Becker Kottwitz Bierhals, C. C., Oliveira Dos Santos, N., Laís Fengler, F., Dellamora Raubustt, K., Ane Forbes, D., \& Giradi Paskulin, L. M. (2017). Needs of familiy caregibers in home care for older adults. Revista Latino-Americana de Enfermagem, 25 , $1-8$. https://www.scielo.br/j/rlae/a/8fvbJZj69yQ9VqTCDNGVG7m/?lang=en\&for mat $=$ pdf

Currie, C., Nottage, C., Spurrier, N., Madden, \& D. (2020). Comparison of public health specialty training in Australia and England. Public Health, 188, 35-41. https://www.sciencedirect.com/science/article/abs/pii/S0033350620303851

Da Silva Viana, I., da Silva, L. F., da Conceição, D. S., Bezerra Goes, F. G., \& Medeiros de Moraes, J. R. (2018). Educational encounter of nursing and the relatives of children with special health care needs. 27(3). https://www.scielo.br/j/tce/a/wPSMMCdMNsg49V6M7gBc6pb/?lang=en

Fan, D., Li, D., Liu, W., Guang, X., \& Boustras, G. (2021). Weaving public health and safety nets to respond the COVID-19 pandemic. Safety Science, 134, 1-8. https://reader.elsevier.com/reader/sd/pii/S0925753520304550?token=1E59F 7546115D10B1AF8FF620CDE50B792F7479DB3381E2866DF1D3CC2686 02A672E00AE4B22A7C4ED44DCADE3A19865\&originRegion=us-east1 \&originCreation $=20211231002009$

Kiernan, C., \& Kilbride, M. (2021). Dying at home of Covid-19: Meeting the need for home-based care. Preventive 145. https://www.sciencedirect.com/science/article/pii/S0091743520304400

Lim, S., Island, L., Horsburgh, A., \& Maier, A. (2021). Home First! Identification of Hospitalized Patients for Home-Based Models of Care. Journal of the American Medical Directors Association, 22, 413-417. 
https://reader.elsevier.com/reader/sd/pii/S1525861020304874?token=4CD78 8E8EE936B065FA2FE79A2FBD332C71BC02A30AB39EBC479081C7A03 0E1036AED11562D09B034BB99875AC40A7D8\&originRegion=us-east1 \&originCreation $=20211230233416$

Maeda, Y., Caskurlu, S., Kenney, R., Kozan, K., \& Richardson, J. (2020). Moving qualitative synthesis research forward in education: A methodological systematic review. Educational Research Review, 35. https://www.sciencedirect.com/science/article/abs/pii/S1747938X21000476

Moreno, B., Muñoz, M., Cuellar, J., Domancic, S., \& Villanueva, J. (2018). Revisiones Sistemáticas: definición y nociones básicas. Periodoncia Implantol. Rehabil, 11(3), 184-186. scielo.cl/pdf/piro/v11n3/0719-0107-piro11-03-184.pdf

Morin, S., Barron, H., Bubb, O., Drehle, C., Lazarus, J., \& Burrone, E. (2021). The economic and public health impact of intellectual property licensing of medicines for low-income and middle-income countries: a modelling study. Public Health, $1-8$. https://reader.elsevier.com/reader/sd/pii/S2468266721002024?token=201AF 9CA4CCA99E1065518BC11E06872DEC90E4131EEBD27729AF69919309 57ED33D68AFD1D1C2DE5555616E652E7FF5\&originRegion=us-east1\&originCreation $=20211230223312$

Munhak da Silva, R. M. (2020). Care opportunities for premature infants: home visits and telephone support. Revista Latino-Americana de Enfermagem, 1-8. https://www.scielo.br/j/rlae/a/jPc4SkMJpHVgRLXtr4XNn4M/?lang=en

Ngan, L. K., Howick, S., McLaffertu, D., Hopkins, G., Pravinkumar, S., Der Meer, R., \& Megiddo, I. (2021). Impact of visitation and cohorting policies to shield residents from covid-19 spread in care homes: an agent-based model. American Journal of Infection Control, 49, 1105-1112. https://reader.elsevier.com/reader/sd/pii/S0196655321004624?token=3FB2C A84039831F69F612C15DA28C35AFC1FC406B7E6B133C9D90EAD3212 D69909370F9E64CB80E24553471057D9A2B8\&originRegion=us-east1\&originCreation $=20211230234546$ 
Pailliard, C., Gautier, L., Degroote, S., Guillard, E., Chabrol, F., \& Ridde, V. (2019). Conceptual analysis of health systems resilience: A scoping review. Social $\begin{array}{llll}\text { Science } \quad \text { Medicine, } & \text { 232, }\end{array}$ https://www.sciencedirect.com/science/article/abs/pii/S0277953619302205

Powers, K., Brandon, J., Townsend, \& Colette. (2021). Preparing nursing students for home health using an escape room: A qualitative study. Nurse Education Today, 108. https://www.sciencedirect.com/science/article/abs/pii/S026069172100472X

Ritchie, C., Gallopyn, N., Sheehan, O., Ahmed, S., Franzosa, E., Gorbenko, K., . . . Leff, B. (2021). COVID Challenges and Adaptations Among Home-Based Primary Care Practices: Lessons for an Ongoing Pandemic from a National Survey. Journal of the American Medical Directors Association, 22, 13381344.

https://reader.elsevier.com/reader/sd/pii/S1525861021004801?token=EFE37 580D35F35B46F7A67A89EF5ABBDE8A2DBFA92D1E3649C7FE04C7A A7049D929145ED6D3E20A1E0A5C1B7A4ABC358\&originRegion=useast-1 \&originCreation $=20211230232256$

Rossetto, V., Gonçalves de Oliveira Toso, B. R., \& Rodriguez, R. M. (2020). Organizational flow chart of home care for children with special health care $\begin{array}{lllll}\text { needs. } & \text { Rev } & \text { Bras }\end{array}$ https://www.scielo.br/j/reben/a/ZkpX6SQFhTTzhDBTMTySh4j/?format=ht ml\&lang=en

Si-qian, Z., Li , Y., Peng-xiang, Z., Hui-bo, L., Fang, L., \& Rong-sheng, Z. (2021). Recommendations and guidance for providing pharmaceutical care services during COVID-19 pandemic: A China perspective. Research in Social and Administrative Pharmacy, 17(1), 1819-1824. https://www.sciencedirect.com/science/article/pii/S1551741120302849

Smith, M., Nordby, P., Menggang, Y., \& Jaffery, J. (2020). A practical model for research with learning health systems: Building and implementing effective complex case management. Applied Ergonomics, 84. https://www.sciencedirect.com/science/article/abs/pii/S0003687019302327 
Staniforth, R., \& Such, E. (2019). Public health practitioners' perspectives of migrant health in an English region. Public Health, 175, 79-86. https://www.sciencedirect.com/science/article/abs/pii/S003335061930215X

Such, E., Laurent, C., Jaipaul, R., \& Salway, S. (2020). Modern slavery and public health: a rapid evidence assessment and an emergent public health approach. Public Health, 180, 168-179. https://www.sciencedirect.com/science/article/abs/pii/S0033350619303385

Videon, T., Rosati, R., \& Landers, S. (2021). COVID-19 infection rates early in the pandemic among full time clinicians in a home health care and hospice organization. American Journal of Infection Control, 50(1), 26-31. https://www.sciencedirect.com/science/article/pii/S019665532100643X

Wanaratwichit, C., Hills, D., \& Cruickshank, M. (2019). Home-based care for people with disabilities: Role of registered nurses within the District health system in Thailand. Collegian, $27(1)$ $18-22$ https://www.sciencedirect.com/science/article/abs/pii/S1322769619300411

Wendt, B., Huisman, G., Bakker, A., Hautvast, J., \& Huis, A. (2021). Exploring infection prevention practices in home-based nursing care: A qualitative observational study. International Journal of Nursing Studies, 125, 1-10. https://reader.elsevier.com/reader/sd/pii/S0020748921002753?token=0FC98 1DFEC8E8DFB8FAC377E0F7674452429EFB75396BFD0F1AF5642FFB5 9A2AC42C4FDE664313C21AE750879D6BACFC\&originRegion=us-east1\&originCreation $=20211230232715$

Yehenew Mengistu, M. P., \& Weihua Sheng, H. (2018). Delivering home healthcare through a Cloud-based Smart Home Environment (CoSHE). Future Generation Computer $\quad$ Systems, 129-140. https://www.sciencedirect.com/science/article/abs/pii/S0167739X17302194

Zhang, X., \& Nie, H. (2021). Public health insurance and pharmaceutical innovation: Evidence from China. Journal of Development Economics, 148. https://www.sciencedirect.com/science/article/abs/pii/S030438782030153X 